Review Article

\title{
Application Areas of Traditional Molecular Genetic Methods and NGS in relation to Hereditary Urological Cancer Diagnosis
}

\author{
Dmitry S. Mikhaylenko $\mathbb{D}^{1},{ }^{1,2}$ Alexander S. Tanas $\mathbb{D D}^{1}$ Dmitry V. Zaletaev $\mathbb{D}^{2}{ }^{2}$ \\ and Marina V. Nemtsova ${ }^{1,2}$ \\ ${ }^{1}$ Laboratory of Epigenetics, Research Centre for Medical Genetics, Moscow 115522, Russia \\ ${ }^{2}$ Laboratory of Medical Genetics, I.M. Sechenov First Moscow State Medical University (Sechenov University), \\ Moscow 119991, Russia \\ Correspondence should be addressed to Dmitry S. Mikhaylenko; dimserg@mail.ru
}

Received 25 March 2020; Revised 22 May 2020; Accepted 3 June 2020; Published 17 June 2020

Academic Editor: Reza Izadpanah

Copyright ( $\odot 2020$ Dmitry S. Mikhaylenko et al. This is an open access article distributed under the Creative Commons Attribution License, which permits unrestricted use, distribution, and reproduction in any medium, provided the original work is properly cited.

\begin{abstract}
Next generation sequencing (NGS) is widely used for diagnosing hereditary cancer syndromes. Often, exome sequencing and extended gene panel approaches are the only means that can be used to detect a pathogenic germline mutation in the case of multiple primary tumors, early onset, a family history of cancer, or a lack of specific signs associated with a particular syndrome. Certain germline mutations of oncogenes and tumor suppressor genes that determine specific clinical phenotypes may occur in mutation hot spots. Diagnosis of such cases, which involve hereditary cancer, does not require NGS, but may be made using PCR and Sanger sequencing. Diagnostic criteria and professional community guidelines developed for hereditary cancers of particular organs should be followed when ordering molecular diagnostic tests for a patient. This review focuses on urological oncology associated with germline mutations. Clinical signs and genetic diagnostic laboratory tests for hereditary forms of renal cell cancer, prostate cancer, and bladder cancer are summarized. While exome sequencing, or, conversely, traditional molecular genetic methods are the procedure of choice in some cases, in most situations, sequencing of multigene panels that are specifically aimed at detecting germline mutations in early onset renal cancer, prostate cancer, and bladder cancer seems to be the basic solution for molecular genetic diagnosis of hereditary cancers.
\end{abstract}

\section{Introduction}

Diagnosis of renal cell cancer (RCC), prostate cancer (PC), and bladder cancer (BC) is an issue in the field of modern urological oncology because of their high incidence among malignant tumors and due to the social significance of these diseases [1]. As with cancers of other organs, solitary sporadic tumors that occur with advancing age account for a majority of urological oncology cases. Only $1 \%$ to $3 \%$ of these cases can be considered manifestations of hereditary cancer syndromes due to germline mutations. However, in many cases, hereditary forms of RCC, PC, and BC are associated with early onset, multiplicity of lesions, and specific nonurological signs, which make identification of germline mutations crucial for final diagnosis [2, 3]. Some hereditary urological cancer syndromes are monogenic diseases caused by point mutations of a single gene, and in some instances, common point mutations observed in a few exons can be diagnosed using relatively inexpensive, routine molecular genetic tests, such as polymerase chain reaction (PCR), multiplex ligation-dependent probe amplification (MLPA), and Sanger sequencing [4]. However, several new causative genes of hereditary urological cancer syndrome caused by germline mutations have recently been discovered via next generation sequencing (NGS) of the genomes and exomes of cancer patients. NGS has shown potential as a useful diagnostic technique when a multiexon candidate gene or several candidate genes must be examined to identify an underlying mutation $[5,6]$. This review characterizes hereditary forms of RCC, PC, and BC (see Table 1) and 
TABLE 1: Main hereditary urological cancer syndromes due to germline mutations.

\begin{tabular}{|c|c|c|}
\hline Disorder (incidence) & Gene & Tumor type \\
\hline $\begin{array}{l}\text { Renal cell cancer } \\
\text { Von Hippel-Lindau syndrome }(1: 40,000) \\
\text { Birt-Hogg-Dube syndrome }(\mathrm{n} / \mathrm{a}) \\
\text { HPRC }(\mathrm{n} / \mathrm{a}) \\
\text { HLRCC }(\mathrm{n} / \mathrm{a}) \\
\text { BAP1-TPDS (n/a) } \\
\text { Hereditary paraganglioma }(1: 1,200,000) \\
\text { Tuberous sclerosis }(1: 6,000-10,000) \\
\text { Other monogenic forms of RCC }(\mathrm{n} / \mathrm{a})\end{array}$ & $\begin{array}{c}V H L \\
F L C N \\
M E T \\
F H \\
B A P 1 \\
S D H A / B / C / D / A F 2 \\
T S C 1 / 2 \\
\text { PBRM1, FHIT:RNF139 }\end{array}$ & $\begin{array}{c}\text { ccRCC } \\
\text { pRCC, chRCC, OC } \\
\text { pRCC type I } \\
\text { pRCC type II } \\
\text { ccRCC } \\
\text { pRCC, ccRCC } \\
\text { AML } \\
\text { ccRCC }\end{array}$ \\
\hline $\begin{array}{l}\text { Prostate cancer } \\
\text { Lynch syndrome in men with PC (n/a) } \\
\text { Hereditary male breast cancer/PC (n/a) }\end{array}$ & $\begin{array}{c}\text { MLH1, MSH2, MSH6, PMS2 } \\
\text { BRCA1/2, CHEK2, ATM }\end{array}$ & $\begin{array}{l}\mathrm{AC} \\
\mathrm{AC}\end{array}$ \\
\hline $\begin{array}{l}\text { Bladder cancer } \\
\text { Lynch syndrome (on average, } 1: 1,000 \text { ) }\end{array}$ & MLH1, MSH2, MSH6, PMS2 & $\mathrm{UC}$ \\
\hline
\end{tabular}

Abbreviations: ccRCC, clear cell renal cell carcinoma; pRCC, papillary renal cell carcinoma; chRCC, chromophobe renal cell carcinoma; OC, oncocytoma; AML, angiomyolipoma; AC, adenocarcinoma; UC, urothelial carcinoma; HPRC, hereditary papillary renal carcinoma type 1; HLRCC, hereditary leiomyomatosis and renal cell carcinoma; BAP1-TPDS, BAP1 tumor predisposition syndrome; n/a, not available.

suggests genetic diagnostic methods for these cases, including those for which balanced application of routine tests is justified and those for which NGS is indicated.

\section{Clinical and Genetic Characteristics of Hereditary Forms of Renal Cancer}

2.1. Von Hippel-Lindau Syndrome. Von Hippel-Lindau (VHL) syndrome (OMIM 193300) is an autosomal dominant hereditary cancer syndrome that occurs at a frequency of one per 39,000 to 90,000 newborns in different populations, with a penetrance $>80 \%$ at the age of 60 [7]. The most common tumors are clear cell RCC (often multifocal and/or bilateral), renal cysts, hemangioblastomas of the central nervous system, retinal angiomas, and pheochromocytoma, whereas neuroendocrine tumors and cysts of the pancreas and endolymphatic sac tumors of the inner ear are far less common [8]. VHL syndrome is caused by mutation of the VHL tumor suppressor gene, which maps to chromosome 3p25 and has three exons and encodes a protein containing 213 amino acid residues. VHL normally binds to CUL2, RBX1, and elongins $\mathrm{B}$ and $\mathrm{C}$ to produce a multiprotein complex that promotes ubiquitin-dependent degradation of hypoxia-inducible factors $1 / 2 \alpha$ (HIF $1 / 2 \alpha)$ [9]. Genotype-phenotype correlations are characteristic of $V H L$ mutations in VHL syndrome, which is classified into type 1 (without pheochromocytoma but with high risk for clear cell RCC) and type 2 (with pheochromocytoma). Type $1 \mathrm{VHL}$ syndrome is associated with frameshifts, nonsense mutations, and missense mutations that prevent the production of mature VHL protein. By contrast, type 2 VHL syndrome is associated with point missense mutations that cluster in regions encoding HIF and the elongin $\mathrm{C}$ binding sites of the VHL protein $[10,11]$. An example of VHL syndrome demonstrates that identification of a causative pathological germline mutation can affect treatment decision. In sporadic kidney tumors, the primary tumor is removed after the completion of diagnostic tests and determination of disease stage. Because the risk of developing multiple tumors, including those in the contralateral kidney, with VHL syndrome is quite high, patients with VHL syndrome confirmed by molecular genetic testing are treated by removing the primary tumor via nephrectomy as soon as the tumor reaches $3 \mathrm{~cm}$ in the largest dimension along with certain contraindications $[12,13]$. However, early metastasis is possible in other hereditary RCC forms, warranting surgery immediately after diagnosis. For example, type II papillary RCC in hereditary leiomyomatosis and RCC (described in the next section of this review) often develops as a solitary unilateral tumor but is characterized by rapid progression [14]. The accumulation of HIF in the cell and, in particular, of its HIF- $2 \alpha$ isoform with oncogenic properties introduces the possibility of therapeutic inhibition of the intermediate pathogenetic pathway triggered by inactivation of VHL. Small synthetic inhibitors have been developed that block the heterodimerization of HIF- $2 \alpha$ with HIF- $1 \beta$ and their DNA binding, thereby disrupting the activation of HIF target genes, as well as drugs that promote VHL-independent HIF degradation. These drugs (panobinostat, entinostat, vorinostat, bortezomib, and others) are used in clinical trials along with other types of targeted therapy in patients with metastatic clear cell RCC [15].

2.2. Hereditary Leiomyomatosis and RCC (HLRCC). HLRCC (OMIM 150800) is associated with multiple leiomyomas (leiomyosarcomas in some cases) of the skin and uterus and RCC and identified in $25 \%$ of HLRCC patients. It is sometimes combined with renal cysts. Renal malignancies in this familial cancer syndrome are often type II papillary carcinomas [16]. Mutations of the fumarate hydratase $(F H)$ tumor suppressor gene are responsible for HLRCC. FH is located on chromosome 1q42 and encodes an enzyme involved in the Krebs cycle. Missense mutations account for $\sim 90 \%$ of all relevant mutations, with no distinct hot spots identified in most populations worldwide (although in some European populations, hot spot mutations were identified in codon 190) [14]. Minor forms of HLRCC syndrome have 
been described. For example, a combination of type II papillary RCC and hemangioblastomas was observed in a patient with a $1.4 \mathrm{Mb}$ deletion, which affected region 1q43, including nine genes in addition to $F H$ [17]. It is worth noting that the role of germline $\mathrm{FH}$ mutations in carcinogenesis is not limited to HLRCC. A case of leiomyomatosis in combination with pheochromocytoma [18] was described, and $F H$ was included in a panel of 10 genes to search for germline pathogenic variants in familial pheochromocytoma [19].

\subsection{Birt-Hogg-Dube Syndrome (BHDS). BHDS (OMIM} 135150 ) is an autosomal dominant syndrome, a major manifestation of which includes multiple fibrofolliculomas. The presence of at least 10 characteristic skin neoplasms, including one histologically identified fibrofolliculoma, and a family history of the disease constitute the minimal diagnostic criteria potentially leading to a preliminary diagnosis of BHDS. Renal tumors, which develop in $35 \%$ of BHDS patients, are usually multifocal and bilateral and belong to different pathomorphological types, with chromophobe carcinomas and hybrid oncocytic/chromophobe tumors being the most common [20,21]. BHDS is caused by germline mutations of folliculin (FLCN), a tumor suppressor gene located on chromosome 17p11.2. Germline FLCN mutations are predominantly loss-of-function mutations, such as frameshifts due to insertions, deletions, and duplications, as well as complex nonsense and splice-site mutations. Missense mutations were identified only in single cases $[22,23]$.

2.4. Hereditary Papillary Renal Carcinoma (HPRC) Type I. HPRC type I (OMIM 605074) is an autosomal dominant disease associated with the development of type I papillary RCCs, which are often multifocal and bilateral. Although inactivation of a tumor suppressor gene is responsible for the three hereditary RCC forms described, germline activating mutations of $M E T$, an oncogene located on chromosome 7q31, causes HPRC [4]. MET germline mutations, observed in HPRC, lead to constitutive activation of the cytoplasmic domain of the receptor and stimulate cell division, constituting the main event in the carcinogenesis of papillary carcinomas in HPRC. Because MET activation is a key event in the HPRC pathogenesis, its therapeutic potential has been suggested for targeted therapy with MET inhibitors (i.e., foretinib, tivantinib, and volitinib) in metastatic HPRC. Studies of cabozantinib and the multikinase inhibitor crizotinib efficacy for papillary RCC treatment are also being carried out $[24,25]$. The targeted MET inhibitor savolitinib led to a four-fold increase in relapse-free survival in papillary RCC with MET mutation [26]. Additionally, promising results have been reported for foretinib for treating metastatic HPRC in 10 patients, with treatment efficacy comparable with or even higher than that in sporadic type I papillary renal cancer. Therefore, MET inhibitors, especially foretinib, might be applicable for targeted therapy of HPRC [27].
2.5. PBRM1 Mutations in Renal Cell Carcinoma. Exome sequencing in sporadic clear cell RCC identified PBRM1, a tumor suppressor gene, as the gene second most often altered by somatic point mutations: $38 \%$ of cases, trailing only VHL (50-60\% of cases) [28]. A cohort of patients with suspected hereditary clear cell RCC and for whom VHL syndrome was excluded was screened for germline PBRM1 mutations. Germline PBRM1 mutations proved to be rare, with the inactivating germline mutation c.3998_4005del identified as the cause of the disease in only one of 35 unrelated candidate families [29].

2.6. The BAP1 Tumor Predisposition Syndrome. According to NGS-based studies, somatic mutations of $B A P 1$ are found in $>10 \%$ of clear cell RCCs. BAP1 encodes a deubiquitinating hydrolase that interacts with the tumor suppressor protein BRCA1. Additionally, BAP1 is considered a tumor suppressor gene, although its functions are still being investigated. Data related to $>180$ families with germline BAP1 mutations have been collected in recent years, enabling identification of target organs, tumor types, and description of the BAP1 tumor predisposition syndrome (BAP1-TPDS, OMIM 614327) as a new hereditary cancer syndrome caused by BAP1 mutations. BAP1-TPDS is mostly associated with mesotheliomas, as well as cutaneous and uveal melanomas, with some patients developing RCC [30].

\subsection{Hereditary Pheochromocytoma-Paraganglioma.}

Hereditary pheochromocytoma-paraganglioma is another rare hereditary cancer syndrome associated with RCC. Paragangliomas occur in 1 of 300,000 people, of which 25\% are associated with germline mutations (i.e., the frequency of the syndrome is $\sim 1: 1.2$ million). This syndrome is caused by germline inactivating mutations of the tumor suppressor genes $S D H A, S D H B, S D H C, S D H D$, and $S D H A F 2$, which encode subunits of the mitochondrial enzyme complex succinate dehydrogenase. In some cases, various pathomorphological types of RCCs caused mostly by $S D H B$ and $S D H D$ mutations might develop [31, 32]. Paragangliomas caused by germline mutations of $S D H B$ can develop not only in the kidneys but also in the bladder. Lack of the SDHB protein according to immunohistochemical (IHC) analysis can be used as a screening test for possible $S D H B$ mutation [33].

2.8. Tuberous Sclerosis. Tuberous sclerosis is a multisystem disease characterized by autosomal dominant inheritance and an incidence of one in 6,000 to 10,000 newborns. The disease exemplifies hereditary renal tumors with genetic heterogeneity and two multiexon candidate genes. Clinical signs of tuberous sclerosis include hypopigmented macules in $95 \%$ of cases and angiofibromas in $40 \%$ to $90 \%$ of cases. Multiple renal angiomyolipomas develop in $80 \%$ of tuberous sclerosis patients [34]. Germline mutations underlying tuberous sclerosis affect the tumor suppressor genes TSC complex subunit (TSC) 1 and TSC2, which encode hamartin and tuberin, respectively [35]. 


\section{Methodological Approaches to Diagnosing Hereditary Forms of Kidney Cancer}

The mission of the oncologist and geneticist in cases of suspected hereditary RCC is to refer the patient to molecular genetic testing to determine the most likely form of RCC and the candidate genes in which to search for a germline mutation. The choice of method for detecting mutations and the testing itself remain with the laboratory molecular diagnostics specialists. However, the choice of coding sequences of genes that should be investigated and, therefore, the choice of mutation analysis method only partially depend on the clinical picture of the disease. Most hereditary forms of RCC listed in the previous section present a characteristic clinical picture and can be considered as monogenic diseases according to the classical manifestation of the syndrome. In such cases, it is possible to search for mutations in only one candidate gene. In particular, in the classic form of VHL syndrome, diagnosis requires PCR amplification and sequencing of the three VHL exons (i.e., three PCR and six Sanger sequencing reactions per sample, which together with the intermediate steps of the analysis is feasible within 2 working days). Such analysis of point mutations allows identification of the cause of the disease in $85 \%$ of families with VHL syndrome [36-38]. In cases of negative results, partial deletions are additionally sought via MLPA or real-time PCR $[39,40]$. However, again using VHL syndrome as an example, pitfalls of traditional molecular genetic methods also exist. For example, a 2C subtype of the syndrome appears only as adrenal pheochromocytoma and requires differential diagnosis with other hereditary cancer syndromes, such as hereditary pheochromocytoma-paraganglioma and type 2 multiple endocrine neoplasia $[31,41,42]$. In this case, it is necessary to examine a panel of six candidate genes. Moreover, although VHL and RET mutations can be detected by sequencing several PCR products, complementation of the testing area with $\mathrm{SDH}$ family genes immediately transfers these diagnostics to the category of tasks that have to be solved not by traditional methods of PCR and Sanger sequencing but by the multigene panel NGS [43].

Until recently, diagnosis of other monogenic forms of RCC was also carried out by PCR and Sanger sequencing because it was a question of sequencing no more than 10 different PCR products. In particular, direct DNA diagnosis of HPRC is based on the identification of missense mutations in MET exons 15 through 21, which encode the cytoplasmic domain of the receptor (7 PCR products) $[12,44]$. PCR amplification and Sanger sequencing of all $10 \mathrm{FH}$ exons enable DNA diagnosis of HLRCC [45]. Genetic laboratory diagnosis of BHDS is based on testing for FLCN mutations via PCR amplification and Sanger sequencing of polypeptide-coding exons 4 through 14 . Note that the $\mathrm{C}_{8}$ mononucleotide tract of exon 11 is a mutation hot spot of FLCN, with germline single-nucleotide deletions/insertions in this tract found in $\sim 25 \%$ to $50 \%$ of affected families (i.e., exon 11 is expediently tested in the search for the underlying mutation) $[46,47]$. In addition to testing for point mutations in
FLCN exons, MLPA is performed to detect deletions, with their frequency in the promoter region of FLCN reported as higher than that in the coding region. Direct sequencing combined with MLPA increased the clinical sensitivity of molecular genetic testing from $80 \%$ to $95 \%$ of BHDS families [48], whereas a lower proportion of patients with verified mutations was reported by other studies [49].

However, in recent years, the search for germline mutations in MET, FLCN, and FH has been increasingly performed using NGS of multigene panels, specifically the TruSight Cancer Panel (Illumina), which includes 94 main oncogenes and tumor suppressor genes [50]. This is due to a decrease in the cost and time of performing NGS tests that has occurred in the previous 10 years, as well as to the similarity of the symptoms and the histological variants of tumors (see Table 1) observed in different forms of hereditary RCC. Basically, Illumina or Ion Torrent platforms with x20-50 reading depths are used to detect germline mutations $[51,52]$. Various reviews in the previous 15 years have focused on comparing equipment cost and productivity, the sequencing price (including the cost of consumables), the percentage of errors in sequenced reads, and other technical characteristics of various NGS platforms $[53,54]$. We note that in many respects, the price of the test and its execution time depend on the capacity of the chips and barcoding used, the frequency of sequencer usage per week (i.e., actually on the number of sequenced libraries of the same type), and the experience of the laboratory specialists in annotating cancerrelated mutations.

Interestingly, the introduction of NGS in studies of the genomes of sporadic tumors and the search for somatic mutations sometimes provide unexpected results relevant to the diagnosis of hereditary cancer syndromes. In one study, MET mutations were detected in type I (presumably sporadic) papillary renal carcinomas, with three of the 17 mutations proving to be germline rather than somatic [55]. This finding indicated that testing for germline MET mutations was justified for younger patients with papillary renal carcinoma type I, even in the absence of multiple primary lesions in the kidneys. The sequencing of multigene panels is especially justified for mutation screening in genes comprising tens of exons. PBRM1 has 10 -fold more exons than $V H L$ and lacks mutation hot spots, thereby warranting the use of NGS to search for mutations. Similar to PBRM1, BAP1 contains 17 exons and lacks recurrent mutations, warranting the utilization of NGS as the optimal technique and through which the pathological mutation might be identified during BAP1-TPDS diagnosis [30]. As noted, all genes of the SDH family must often be checked for mutations in order to diagnose hereditary pheochromocytomaparaganglioma; therefore, exome sequencing or testing via proper gene panels is the appropriate method for direct DNA diagnosis of this syndrome [43]. TSC1 contains 23 exons, and TSC2 contains 42, with the relevant mutations distributed throughout the coding regions in a regular manner and including nonsense, missense, and splice-site mutations, as well as short insertions/deletions and extended deletions. Therefore, because these genes are large in size and 
lack mutation hot spots, NGS is warranted as the main method for potentially identifying causes of this syndrome. When confronted with negative sequencing results, MLPA is employed for expedient testing of extended deletions or duplications. A combination of these methods enables effective detection of germline mutations of TSC1 and TSC2 in $80 \%$ to $90 \%$ of tuberous sclerosis patients $[56,57]$.

At the same time, the larger the multigene panel, the more increased the number of identified genetic variants potentially difficult to classify in terms of clinical relevance, with this problem even more pronounced for exome sequencing. The guidelines of professional cancer associations do not currently include exome sequencing as an element of the diagnostic algorithms for RC, PC, or BC; therefore, it should be considered as an additional test with limited diagnostic value.

Finally, in the absence of criteria for known hereditary cancer syndromes but with a family history of the disease, early manifestation, and/or multiple tumors, the use of exome sequencing to search for pathogenic germline mutations might well be justified. This enables possible determination of a causative mutation in the minor candidate gene not described earlier or identification of combinations of germline mutations in several of these genes in families with a history of renal carcinoma. For example, sequencing of an exome from siblings in a family with papillary thyroid cancer and clear cell RCC revealed a combination of the SDHA heterozygous mutation along with mutations in the TGFB2 and PARP1 genes [58]. In another family, hereditary renal carcinoma with areas of angioleiomyomatous stroma was described as an atypical variant of the manifestation of the TSC2 missense mutation [59]. Therefore, preliminary diagnosis and selection of candidate genes to search for mutations depend on the classical or, by contrast, atypical manifestation of hereditary RCC. This, in turn, determines the choice of a multigene panel for NGS, exome sequencing, and, in rare cases, traditional methods for the identification of point mutations and gross deletions.

\section{Molecular Genetic Diagnostics of Hereditary Prostate Cancer}

PC manifests as a hereditary cancer syndrome due to germline mutations in only $1 \%$ to $2 \%$ of cases. Carriers of $B R C A 1$ and $B R C A 2$ mutations are at high risk of developing PC, with $B R C A 2$ mutations contributing more to hereditary PC than BRCA1 mutations [60, 61]. Lynch syndrome (incidence, 1 in 370-1,500 people in European and American populations), which is caused by mutations in the DNAmismatch repair (MMR) genes MLH1, MSH2, MSH6, and $P M S 2$, manifests with early-onset $\mathrm{PC}$ in some cases. In contrast to the manifestation of the classical form of Lynch syndrome as a hereditary nonpolyposis colorectal cancer, in hereditary PC, germline mutations are observed mainly in the MSH2 and MSH6 genes and much less often in MLH1. The proportion of $\mathrm{MSH} 2$ mutations in such patients is up to $79 \%$ of cases. However, PC is not among the frequent tumors observed in Lynch syndrome (among 6,350 carriers of MMR gene mutations in a mixed cohort with an approximately equal ratio of both sexes, PC was diagnosed in 26 men). The risk of developing this cancer due to MMR gene mutations is only three- to five-fold higher, and often the first clinical manifestation of the disease and the cause of death are related to colorectal cancer, which can lead to an underestimation of the incidence of $\mathrm{PC}$ in patients carrying germline mutations in $\mathrm{MSH} 2 / 6$ [62-64]. In addition to the MMR genes, the c.251G > A (p.G84E) mutation in HOXB13 associated with a 20 -fold increase in the relative risk for PC [65]. Other genes that are considered associated with hereditary PC include TP53, NBN, BRIP1, and other DNArepair genes [66]. In general, according to the recommendations of the National Comprehensive Cancer Network (NCCN; v.2.2019), it is preferable to use NGS to sequence the multigene panel, which includes at least MMR genes mutated in Lynch syndrome and a number of other DNArepair genes (BRCA2, BRCA1, ATM, CHEK2, PALB2, MLH1, MSH2, MSH6, and PMS2). This panel can be supplemented with other candidate genes [67]. For example, sequencing of 94 oncogenes and tumor suppressor genes was performed in 121 cases with early PC onset or a family history of the disease, resulting in pathogenic and likely pathogenic genetic variants observed in $14 \%$ of the cases. Thirteen patients harbored mutations in genes known to be candidates for initiating hereditary PC, whereas the others carried single mutations of genes not previously considered candidates for hereditary PC [68]. If minor candidate genes are excluded, then data from related studies indicate that $8 \%$ of young PC patients have hereditary PC and that a diagnostic panel for hereditary PC should include at least BRCA1, BRCA2, ATM, BRIP1, CHEK2, NBN (c.657del5), HOXB13 (p.G84E), MLH1, MSH2, MSH6, and PMS2 [69].

The categories of patients to whom molecular genetic testing should be recommended if hereditary PC is suspected were defined in recommendations made in the previous 3 to 5 years. In particular, criteria from a Johns Hopkins group included at least three first-line relatives with PC or cases of PC in three generations, as well as two relatives with PC and $\leq 55$ years of age. The indications for testing provided by the American College of Medical Genetics in the first paragraph coincided with those from the Johns Hopkins group (three or more first-line relatives of patients with PC) and included the following assignments to the risk group: people with two or more first-degree relatives who were diagnosed with PC and $\leq 55$ years of age; and PC with a Gleason score $>7$ and at least two relatives with breast, ovarian, or pancreatic cancer. Additionally, the Gleason score was considered significant starting with the previous version of the recommendations of the NCCN, which included three criteria for testing advisability: (1) PC with Gleason score $\geq 7$ and at least one close relative $\leq 50$ years of age with $\mathrm{BC}$ and/or invasive ovarian cancer; (2) PC with a Gleason score $\geq 7$ and relatives with $\mathrm{PC}$ also with a Gleason score $\geq 7, \mathrm{BC}$, and/or pancreatic cancer at any age; and (3) patients with primary metastatic PC $[69,70]$. Among patients with primary aggressive tumors, as well as those with castration-resistant PC, pathogenic germline variants are more likely to be found than on average in PC, which explains the association with initially high Gleason scores. Nevertheless, the principal criteria for 
diagnosing hereditary PC remain as follows: PC at a younger age (up to 55 years), family members with confirmed germline $B R C A 1$ and $B R C A 2$ mutations, or presenting signs of hereditary cancer syndromes associated with mutations in DNA-repair genes [70-72]. In the current version of the NCCN recommendations (v.2.2019), the following indications for testing are defined: patients with a family history of the disease (consonant with the 2018 version and with the addition of specific signs of hereditary cancer syndromes), intraductal histological type PC, intermediate- or high-risk primary $\mathrm{PC}$, locally advanced or metastatic primary PC, and ethnic background as Ashkenazi Jew [67]. In the latter case, the recommendations refer to a population with major mutations. Summarizing these criteria, most authors suggest a search for germline mutations in patients with PC and one of the following criteria: age $<55$ years; immediate relatives with PC; family history of already identified BRCA1/2 mutations or breast, ovarian, or pancreatic cancer; and PC with Gleason score $>7$ in the presence of signs of hereditary PC according to NCCN guidelines [70].

There might be exceptions to this approach for certain populations, in which several major mutations are present due to the founder effect. In these cases, NGS diagnosis can be performed for patients with a negative PCR test for major mutations. In particular, this applies to Ashkenazi Jews, who have three major mutations: c.68_69del, c.5266dupC (BRCA1), and c.5946del (BRCA2). The proportion of their carriers among all tested patients is $\sim 20 \%$. In patients without major mutations, NGS can detect the pathogenic $B R C A 1$ and BRCA2 mutation in another $8 \%$ of cases $[73,74]$. Another example is the increased frequency of the c.5266dupC mutation (referred to as 5382insC in the cited publications) in the BRCA1 gene in European part of Russia, which is responsible for $10 \%$ to $17 \%$ of cases of breast and ovarian cancer $[75,76]$. The same phenomenon is also observed in some Eastern European countries with a predominantly Slavic population neighboring Russia, in particular in Ukraine [77] and Belarus [78]. Comparing the cost of two-stage testing in these populations against a general NGS-based approach, the cost of targeted NGS panels for the diagnosis of hereditary PC per analysis for one patient ranges from $\$ 250$ to $\$ 1,500$, depending on how many loci are included in the panel in addition to BRCA1/2 [69]. Because the proportion of the 5382 ins $C$ mutation among patients in East Slavic populations can exceed 50\%, a simple and cheap $(<\$ 100)$ test based on real-time PCR would allow determination of the major BRCA1 mutation in half of the tested patients within 1 day $[79,80]$ (unfortunately, a significant proportion of publications on this subject do not have an English version). However, we emphasize that such a twostage search for a pathogenic mutation in the BRCA1/2 genes can be performed only with sufficient clinical, genealogical, and population justification. In general, the main strategy for mutation search remains the use of NGS for sequencing the coding parts of hereditary PC candidate genes.

Identification of the germline BRCA1/2 mutation has not only diagnostic but also prognostic significance. Patients with $B R C A 1 / 2$ mutations (germline or somatic) respond better to therapy with drugs that inhibit the remaining alternative DNA-repair pathway involving PARP inhibitors, as well as with platinum-based anticancer drugs. In particular, $88 \%$ of patients with $B R C A 1 / 2$ mutations respond to olaparib PARP inhibitor therapy versus $6 \%$ among those without identified mutations. The PD-1 inhibitor pembrolizumab, an immune-checkpoint inhibitor, is used for cases with severe microsatellite instability (MSI), regardless of the type of tumor, and might be prescribed to patients with Lynch syndrome [81, 82].

\section{Lynch Syndrome as a Form of Hereditary Bladder Cancer}

In the majority of cases, diagnosis of hereditary $\mathrm{BC}$ is reduced to diagnosing urothelial carcinoma as a part of the clinical picture of Lynch syndrome. Lynch syndrome is a hereditary cancer syndrome that usually manifests at a younger age as nonpolyposis colorectal cancer. For example, urothelial carcinoma, which is the second most frequent minor tumor after endometrioid carcinoma, has been detected in 75 of 1,624 Lynch syndrome patients [83]. As noted, Lynch syndrome is caused by germline inactivating mutations of tumor suppressor genes encoding the main components of the MMR system. The frequencies of mutations of these genes in total in all clinical forms of Lynch syndrome are $35 \%$ to $45 \%$ (MLH1 and MSH2), $5 \%$ to $10 \%$ (MSH6), and $~ 5 \%$ (PMS2). Detection of MSI in the tumor is used as a relatively simple screening test to genetically diagnose Lynch syndrome. Another screening test is IHC analysis with an antibody panel that can detect the lack of expression of DNA-repair factors MLH1, MSH2, MSH6, and PMS2. Concordance of results of the molecular genetic MSI test and IHC detection of these factors reaches $94 \%$, which allows their consideration as almost equivalently effective methods for the screening of Lynch syndrome [84]. MSI and IHC have their own peculiarities of practical application. In particular, a positive MSI result in the diagnosis of Lynch syndrome is established when $>30 \%$ of the studied short tandem repeat markers show the presence of aberrant alleles (MSI-High), whereas the absence of aberrant alleles (MSILow) is considered a negative result. The number of identified unstable loci depends on the panel of microsatellites; therefore, it is recommended to use mononucleotide rather than dinucleotide markers and at least five loci. IHC enables the identification of which repair factor is absent; however, the problem of evaluating samples with an intermediate level of staining remains. Therefore, the choice of MSI versus IHC depends more upon which method is more routine in the laboratory: PCR and fragment analysis on a capillary genetic analyzer or a stream of IHC tests using automated systems, such as Ventana $[85,86]$. It should be kept in mind that both MSI and IHC are only screening tests for Lynch syndrome and that germline mutation testing is still required to confirm the diagnosis.

Mutations of MSH2 are four-fold more frequent in Lynch syndrome with BC than mutations of $M L H 1$, the candidate gene most often affected in Lynch syndrome (up to $20 \%$ of patients harboring $\mathrm{MSH} 2$ mutations have $\mathrm{BC}$ ) $[63,87]$. NGS enables a pathological mutation to be 
identified by sequencing the coding regions of candidate genes for Lynch syndrome and other hereditary BC forms using comprehensive cancer gene panels or small customized panels designed for the disease. The fact that $\mathrm{MSH} 2$ mutations are more frequent than mutations of other genes in BC might be important for selecting a less expensive NGS protocol and using panels for targeted candidate gene sequencing in Lynch syndrome [88].

\section{Panels for NGS-Based Diagnostics of Hereditary Forms of Urological Cancers}

Patients with some of the urological cancer syndromes would benefit from an NGS-based search for causative germline mutations. The first variant of an NGS-based approach utilizes gene panels selected via a literature survey of candidate genes for hereditary urological cancers. For example, sequencing of a panel of 23 genes in young RCC patients revealed pathogenic germline variants in 10 genes in $9.5 \%$ of the patients [6]. Another study used the targeted panel designed for hereditary RCC, which includes 19 genes: BAP1, FH, FLCN, MET, MLH1, MSH2, MSH6, MITF, PMS2, EPCAM, PTEN, SDHA, SDHB, SDHC, SDHD, TP53, TSC1, TSC2, and VHL. Pathogenic genetic variants were identified in $6.1 \%$ of 1,235 patients with suspected hereditary cancers of various organs, and among the examined cases, $43.7 \%$ with available submitted histology were consistent with published literature based on the specific gene alteration. Moreover, for patients who provided sufficient personal and family history, only $32.9 \%$ had a strong suspicion for the identified gene alteration. This suggests that a significant proportion of cases with nonclassical manifestation of hereditary RCC might be false negative according to the results of the study of only one candidate gene [89]. Another approach is the use of premade panels of major oncogenes and tumor suppressor genes involved in the carcinogenesis of various tumors and offered as ready-to-use solutions by major manufacturers of equipment and reagents for NGS. Small 50-gene and an extended AmpliSeq panel of 409 genes are offered by Thermo Fisher Scientific and Illumina $[90,91]$. Premade panels have the advantage of being reliable and available but are designed to test for a broad spectrum of common hereditary cancer syndromes and not specific for hereditary urological cancers. The total gene list contains only the 20 genes listed here as the main candidates for initiating hereditary urological oncology diseases (see Figure 1). Evidently and for the purposes of urological oncology, there are 45 unnecessary genes in the small panel and 392 in the large one. These findings suggest the use of multigene panels designed specifically for the diagnosis of hereditary urological cancer syndromes as the most effective diagnostic NGS approach.

Analysis of germline genetic variants obtained by NGS inevitably encounters the following issue: apart from mutations with proven clinical significance and likely pathogenic variants, as well as those that are benign or likely benign, there exists a set of variants with uncertain clinical significance (VUS) [92]. If a VUS is detected as a result of analyzing sequencing data obtained for a patient with

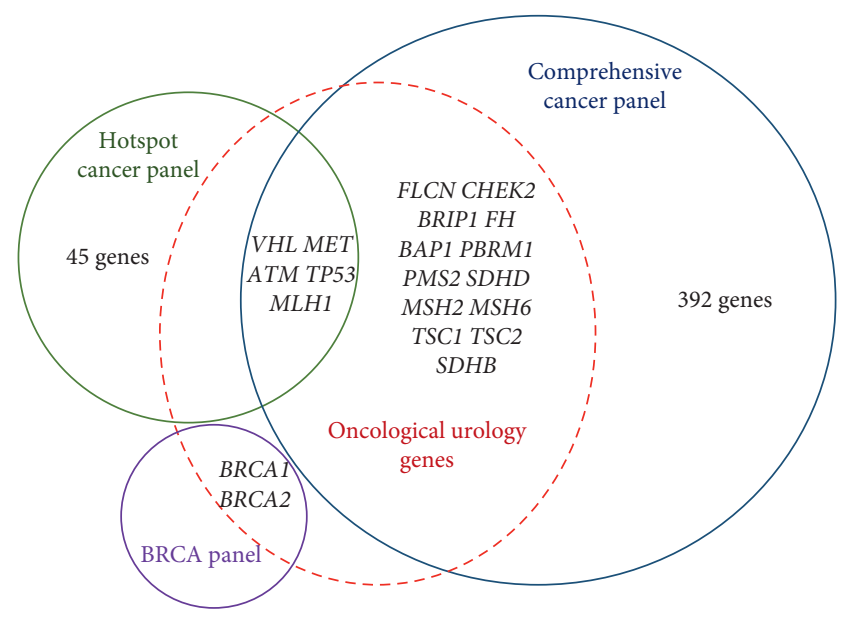

FIgURE 1: Major candidate genes for hereditary urological cancer disorders in ready-to-use AmpliSeq panels for targeted sequencing. The Venn diagram shows differences and similarities in various AmpliSeq panels. The blue circle includes genes comprising the comprehensive cancer panel, the green circle includes genes in the cancer hotspot panel, and the purple circle represents the BRCA panel. The dashed red boundary surrounds the gene mutations responsible for the development of the most common hereditary urological cancer diseases.

suspected hereditary RC, PC, or BC and in the absence of pathogenic/likely pathogenic mutations, this would require a more in-depth analysis and a balanced approach to medical genetic counselling [93]. In general, primary analysis of genetic variants detected by sequencing, in particular, of AmpliSeq panels on the Ion Torrent platform is carried out using Torrent Variant Caller. For visual data analysis and manual filtration of artifacts, the Integrative Genomics Viewer (http://software.broadinstitute.org/software/igv/) is used, and identified genetic variants are annotated using ANNOVAR software (https://annovar.openbioinformatics. org/en/latest/user-guide/download/). For variant annotation, databases of human germline mutations and polymorphisms (i.e., ClinVar, HGMD, and LOVD) are used, as well as databases focused on a specific disease/gene (i.e., Consortium of Investigators of Modifiers of BRCA1/2CIMBA). Significant factors in the analysis of pathogenicity include mutant allele frequency (MAF) and the conservatism of the variable codon. A MAF $<0.01$ and the presence of an amino acid change occurring in a highly conserved site represent a pathogenicity factor in the identified genetic variant $[94,95]$. If there is no information on the role of a germline VUS in carcinogenesis, it is possible that data on identical somatic driver mutations are available in the COSMIC database and the Cancer Genome Atlas [96]. As a rule, VUSs include missense variants, deletions/insertions without frameshifts, and nucleotide substitutions at splice sites, with the majority representing missense variants [97]. Furthermore, VUSs can be analyzed both in silico and in vitro.

In the first case, the effect of a mutation on protein function and prediction of the pathogenicity of novel missense mutations can be evaluated using PolyPhen2, 
MutationTaster, SIFT, and other predictors [98]. Collation with clinical data and the results of other laboratory tests can also help reclassify VUSs. For example, in the diagnosis of Lynch syndrome, co-segregation with colorectal cancer in the pedigree and MSI-High status of the tumor of the proband indicate the pathogenic nature of the detected genetic variant [97]. In the second case, with appropriate laboratory facilities available, functional tests are used to analyze the pathogenicity of the novel mutation. In particular, for VUS analysis in BRCA1, the homology-directed recombination assay is used. In this approach, the VUS is inserted into the expressed vector introduced into HeLa-DR cells, and the expression of endogenous BRCA1 is inhibited by small-interfering RNA. These cells are then transfected with a vector expressing an endonuclease that introduces DNA breaks. If the VUS is pathogenic, the cells will not be able to synthesize functionally active BRCA1 and perform effective DNA repair. As a result, cells undergo apoptosis and exhibit decreased fluorescence [99]. Functional tests also include the methylation tolerance assay, which allows reclassification of novel alleles in $M S H 2$ and $M L H 1$, where the proportion of VUSs in patients with suspected Lynch syndrome can reach $30 \%$. This allows expression of the allele in question in MSH2- or MLH1-deficient cell lines along with subsequent exposure to methylating agents. Normally, activity of MMR proteins should promote the death of cells harboring multiple modified noncomplementary bases; therefore, a significant percentage of surviving cells indicates impaired $M S H 2 / M L H 1$ function and VUS pathogenicity [100]. If tumor material is available for laboratory analysis and the VUS is localized in the tumor suppressor gene, the selective deletion of the reference homologous allele in the tumor DNA can be considered a loss of heterozygosity according to the Knudsen theory. This allows the reclassification of the VUS, thereby increasing pathogenicity score (as shown by the example involving the BRCA2 gene) [101].

The abundance of VUSs detected within multigene panels and their even greater number in exome-sequencing output have hampered the use of genome or exome sequencing as a diagnostic tool in clinical practice, despite the examples of mutation detection by whole-exome sequencing. Errors in interpreting genetic variants are predominantly associated with VUSs $(p<0.001$, comparing the five classes of genetic variants) [93]. Additionally, the fraction of VUSs is higher in the populations of Africa, Latin America, Asia, and Oceania than in Caucasians, for whom more results of genetic studies have been published [102]. Moreover, the fraction of VUSs among all identified germline variants can be quite high for multigene panels (i.e., $37 \%$ for a panel of 12 candidate genes for hereditary PC) [71]. Furthermore, in silico testing is limited, and highly reliable functional tests are often time consuming and laborious, thereby limiting their use for molecular genetic testing in oncology. Until recently, the main NGS approach remained the sequencing of gene panels with an increasing number of published approaches that allow reclassification of VUSs in the diagnosis of hereditary RCC, PC, and BC.

\section{Conclusions}

Urological oncology disorders caused by germline mutations constitute a broad set of oncology diseases, some of which are highly heterogeneous both clinically and genetically. Some of these are caused by mutations of one candidate gene and/or exhibit specific characteristics, including nonurological manifestations (VHL syndrome, BHDS, HLRCC, and HPRC), whereas other hereditary cancer syndromes are due to mutations that might affect any site in the coding region of one candidate gene (BAP1TPDS) or one of the several genes (tuberous sclerosis, hereditary PC with BRCA1/2 mutations, and Lynch syndrome with BC) consisting of $>10$ exons. In such cases, it is necessary to sequence the coding regions of all candidate genes. Premade target sequencing cancer panels include genes that are not directly related to hereditary urological cancer syndromes, and such genes account for $90 \%$ to $96 \%$ of all panel genes. In general, it is possible to formulate the main diagnostic approaches to identifying a pathogenic germline mutation that causes hereditary RCC, PC, and BC. In rare cases of the classic manifestation of a monogenic disease and/or the existence of major mutations in the population (VHL syndrome with a candidate gene of three short exons, BRCA1/2 mutations in Ashkenazi Jews and Eastern Slavs, and an MSI test for Lynch syndrome), analysis is possible, at least in the first stage, using classical methods, such as PCR, Sanger sequencing, and MLPA. All other situations require the use of NGS. In these cases, targeted sequencing of the coding regions of tens of known candidate genes for hereditary oncological diseases is effective. The first approach with NGS is the sequencing of precast panels comprising from several tens to a broad panel of genes known to cause hereditary cancer syndromes. An advantage of this approach is the sequencing of most candidate genes for hereditary cancer syndromes, the constantly decreasing cost of the test, and the reliability of the analytical process in genetic laboratories. The disadvantages are a large number of VUSs and, consequently, difficulties in further medical and genetic counselling. Moreover, not all candidate genes for hereditary oncological diseases are included in the NGS panels. An alternative approach would be to use limited panels that include a couple of dozen genes. The results of sequencing such a panel would be easier to annotate and interpret for counselling, especially if the laboratory and the consulting doctors are focused on the diagnosis of hereditary cancer syndromes. However, there is a risk of omitting a pathogenic mutation in a minor candidate gene not included in such a limited panel. Therefore, the sequencing of multigene panels appears to be the basic solution for molecular genetic diagnosis of hereditary urological cancers.

\section{Disclosure}

This research was carried out within the state assignment of the Russian Federation. 


\section{Conflicts of Interest}

The authors declare that there are no conflicts of interest regarding the publication of this paper.

\section{References}

[1] F. Bray, J. Ferlay, I. Soerjomataram, R. L. Siegel, L. A. Torre, and A. Jemal, "Global cancer statistics 2018: GLOBOCAN estimates of incidence and mortality worldwide for 36 cancers in 185 countries," CA: A Cancer Journal for Clinicians, vol. 68, no. 6, pp. 394-424, 2018.

[2] J. M. Kennedy, X. Wang, K. R. Plouffe et al., "Clinical and morphologic review of 60 hereditary renal tumors from 30 hereditary renal cell carcinoma syndrome patients: lessons from a contemporary single institution series," Medical Oncology, vol. 36, no. 9, p. 74, 2019.

[3] H. T. Lynch, O. Kosoko-Lasaki, S. W. Leslie et al., "Screening for familial and hereditary prostate cancer," International Journal of Cancer, vol. 138, no. 11, pp. 2579-2591, 2016.

[4] E. R. Maher, "Hereditary renal cell carcinoma syndromes: diagnosis, surveillance and management," World Journal of Urology, vol. 36, no. 12, pp. 1891-1898, 2018.

[5] R. Kamps, R. Brandão, B. Bosch et al., "Next-generation sequencing in oncology: genetic diagnosis, risk prediction and cancer classification," International Journal of Molecular Sciences, vol. 18, no. 2, p. 308, 2017.

[6] J. Wu, H. Wang, C. J. Ricketts et al., "Germline mutations of renal cancer predisposition genes and clinical relevance in Chinese patients with sporadic, early-onset disease," Cancer, vol. 125, no. 7, pp. 1060-1069, 2019.

[7] A. N. A. van der Horst-Schrivers, W. J. Sluiter, R. C. Kruizinga et al., "The incidence of consecutive manifestations in Von Hippel-Lindau disease," Familial Cancer, vol. 18, no. 3, pp. 369-376, 2019.

[8] J. Findeis-Hosey, K. McMahon, and S. Findeis, "Von HippelLindau disease," Journal of Pediatric Genetics, vol. 5, no. 2, pp. 116-123, 2016.

[9] N. Varshney, A. A. Kebede, H. Owusu-Dapaah, J. Lather, M. Kaushik, and J. S. Bhullar, "A review of von HippelLindau syndrome," Journal of Kidney Cancer and VHL, vol. 4, no. 3, pp. 20-29, 2017.

[10] I. Ben-Skowronek and S. Kozaczuk, "Von Hippel-Lindau syndrome," Hormone Research in Paediatrics, vol. 84, no. 3, pp. 145-152, 2015.

[11] J. Crespigio, L. C. L. Berbel, M. A. Dias et al., "Von HippelLindau disease: a single gene, several hereditary tumors," Journal of Endocrinological Investigation, vol. 41, no. 1, pp. 21-31, 2018.

[12] M. I. Carlo, A. A. Hakimi, G. D. Stewart et al., "Familial kidney cancer: implications of new syndromes and molecular insights," European Urology, vol. 76, no. 6, pp. 754-764, 2019.

[13] W. M. Linehan, L. S. Schmidt, D. R. Crooks et al., "The metabolic basis of kidney cancer," Cancer Discovery, vol. 9, no. 8, pp. 1006-1021, 2019.

[14] L. Schmidt and W. M. Linehan, "Hereditary leiomyomatosis and renal cell carcinoma," International Journal of $\mathrm{Ne}$ phrology and Renovascular Disease, vol. 7, pp. 253-260, 2014.

[15] O. Martínez-Sáez, P. Gajate Borau, T. Alonso-Gordoa, J. Molina-Cerrillo, and E. Grande, "Targeting HIF-2 $\alpha$ in clear cell renal cell carcinoma: a promising therapeutic strategy," Critical Reviews in Oncology/Hematology, vol. 111, pp. 117-123, 2017.
[16] S. L. Skala, S. M. Dhanasekaran, and R. Mehra, "Hereditary leiomyomatosis and renal cell carcinoma syndrome (HLRCC): a contemporary review and practical discussion of the differential diagnosis for HLRCC-associated renal cell carcinoma," Archives of Pathology \& Laboratory Medicine, vol. 142, no. 10, pp. 1202-1215, 2018.

[17] E. Dow and I. M. Winship, "Hemangioblastoma in hereditary leiomyomatosis and renal cell cancer syndrome: a phenotypic overlap between VHL and HLRCC syndromes," Familial Cancer, vol. 18, no. 1, pp. 91-95, 2019.

[18] S. Richter, L. Gieldon, Y. Pang et al., "Metabolome-guided genomics to identify pathogenic variants in isocitrate dehydrogenase, fumarate hydratase, and succinate dehydrogenase genes in pheochromocytoma and paraganglioma," Genetics in Medicine, vol. 21, no. 3, pp. 705-717, 2019.

[19] E. Sbardella, T. Cranston, A. M. Isidori et al., "Routine genetic screening with a multi-gene panel in patients with pheochromocytomas," Endocrine, vol. 59, no. 1, pp. 175-182, 2018.

[20] L. S. Schmidt, M. L. Nickerson, M. B. Warren et al., "Germline BHD-mutation spectrum and phenotype analysis of a large cohort of families with Birt-Hogg-dubé syndrome," The American Journal of Human Genetics, vol. 76, no. 6, pp. 1023-1033, 2005.

[21] P. R. Benusiglio, S. Giraud, S. Deveaux et al., "Renal cell tumour characteristics in patients with the Birt-Hogg-Dubé cancer susceptibility syndrome: a retrospective, multicentre study," Orphanet Journal of Rare Diseases, vol. 9, no. 1, p. 163, 2014.

[22] L. S. Schmidt and W. M. Linehan, "FLCN: the causative gene for Birt-Hogg-Dubé syndrome," Gene, vol. 640, pp. 28-42, 2018.

[23] L. S. Schmidt and W. M. Linehan, "Clinical features, genetics and potential therapeutic approaches for Birt-Hogg-Dubé syndrome," Expert Opinion on Orphan Drugs, vol. 3, no. 1, pp. 15-29, 2015.

[24] Y. Miyata, A. Asai, K. Mitsunari et al., "Met in urological cancers," Cancers, vol. 6, no. 4, pp. 2387-2403, 2014.

[25] G. Courthod, M. Tucci, M. Di Maio, and G. V. Scagliotti, "Papillary renal cell carcinoma: a review of the current therapeutic landscape," Critical Reviews in Oncology/Hematology, vol. 96, no. 1, pp. 100-112, 2015.

[26] K. Ito, "Recent advances in the systemic treatment of metastatic non-clear cell renal cell carcinomas," International Journal of Urology, vol. 26, no. 9, pp. 868-877, 2019.

[27] K. E. Smith and M. A. Bilen, "A review of papillary renal cell carcinoma and MET inhibitors," Kidney Cancer, vol. 3, no. 3, pp. 151-161, 2019.

[28] W. M. Linehan and C. J. Ricketts, "The cancer genome atlas of renal cell carcinoma: findings and clinical implications," Nature Reviews Urology, vol. 16, no. 9, pp. 539-552, 2019.

[29] P. R. Benusiglio, S. Couvé, B. Gilbert-Dussardier et al., "A germline mutation in PBRM1 predisposes to renal cell carcinoma," Journal of Medical Genetics, vol. 52, no. 6, pp. 426-430, 2015.

[30] S. Walpole, A. L. Pritchard, C. M. Cebulla et al., "Comprehensive study of the clinical phenotype of germline $B A P_{1}$ variant-carrying families worldwide," Journal of the National Cancer Institute, vol. 110, no. 12, pp. 1328-1341, 2018.

[31] G. Wang and P. Rao, "Succinate dehydrogenase-deficient renal cell carcinoma: a short review," Archives of Pathology \& Laboratory Medicine, vol. 142, no. 10, pp. 1284-1288, 2018.

[32] B. Bausch, F. Schiavi, Y. Ni et al., "Clinical characterization of the pheochromocytoma and paraganglioma susceptibility genes SDHA, TMEM127, MAX, and SDHAF2 for gene- 
informed prevention," JAMA Oncology, vol. 3, no. 9, pp. 1204-1212, 2017.

[33] A. Giubellino, K. Lara, V. Martucci et al., "Urinary bladder paragangliomas," The American Journal of Surgical Pathology, vol. 39, no. 11, pp. 1488-1492, 2015.

[34] M. P. Islam and E. S. Roach, "Tuberous sclerosis complex," Neurocutaneous Syndromes, vol. 132, pp. 97-109, 2015.

[35] H. Pópulo, J. M. Lopes, and P. Soares, "The mTOR signalling pathway in human cancer," International Journal of Molecular Sciences, vol. 13, no. 2, pp. 1886-1918, 2012.

[36] P. Chittiboina and R. R. Lonser, "Von Hippel-Lindau disease," Neurocutaneous Syndromes, vol. 132, pp. 139-156, 2015.

[37] A. R. Webster, E. R. Maher, and A. T. Moore, "Clinical characteristics of ocular angiomatosis in von Hippel-Lindau disease and correlation with germline mutation," Archives of Ophthalmology, vol. 117, no. 3, pp. 371-378, 1999.

[38] A. Rasmussen, S. Nava-Salazar, P. Yescas et al., "Von HippelLindau disease germline mutations in Mexican patients with cerebellar hemangioblastoma," Journal of Neurosurgery, vol. 104, no. 3, pp. 389-394, 2006.

[39] H.-J. Cho, C.-S. Ki, and J.-W. Kim, "Improved detection of germline mutations in Korean VHL patients by multiple ligation-dependent probe amplification analysis," Journal of Korean Medical Science, vol. 24, no. 1, pp. 77-83, 2009.

[40] A. Ebenazer, S. Rajaratnam, and R. Pai, "Detection of large deletions in the VHL gene using a real-time PCR with SYBR green," Familial Cancer, vol. 12, no. 3, pp. 519-524, 2013.

[41] C. Eng, "Multiple endocrine neoplasia type 2," in GeneReviews ${ }^{\circledR}$, M. P. Adam, H. H. Ardinger, R. A. Pagon et al., Eds., University of Washington, Seattle, Seattle, WA, USA, 2020.

[42] S. Glasker, H. P. Neumann, C. A. Koch et al., Endotext, MDText.com, Inc., South Dartmouth, MA, USA, 2018.

[43] P. Liu, M. Li, X. Guan et al., "Clinical syndromes and genetic screening strategies of pheochromocytoma and paraganglioma," Journal of Kidney Cancer and VHL, vol. 5, no. 4, pp. 14-22, 2018.

[44] K. A. W. Wadt, A.-M. Gerdes, T. V. O. Hansen, B. G. Toft, L. Friis-Hansen, and M. K. Andersen, "Novel germline c-MET mutation in a family with hereditary papillary renal carcinoma," Familial Cancer, vol. 11, no. 3, pp. 535-537, 2012.

[45] V. M. Raymond, C. M. Herron, T. J. Giordano, and S. B. Gruber, "Familial renal cancer as an indicator of hereditary leiomyomatosis and renal cell cancer syndrome," Familial Cancer, vol. 11, no. 1, pp. 115-121, 2012.

[46] X. Hou, Y. Zhou, Y. Peng et al., "Birt-Hogg-Dubé syndrome in two Chinese families with mutations in the FLCN gene," BMC Medical Genetics, vol. 19, no. 1, p. 14, 2018.

[47] E. C. Sattler, M. Reithmair, and O. K. Steinlein, "Kidney cancer characteristics and genotype-phenotype-correlations in Birt-Hogg-Dubé syndrome," PLoS One, vol. 13, no. 12, Article ID e0209504, 2018.

[48] J. N. Benhammou, C. D. Vocke, A. Santani et al., "Identification of intragenic deletions and duplication in the FLCN gene in Birt-Hogg-Dubé syndrome," Genes, Chromosomes and Cancer, vol. 50, no. 6, pp. 466-477, 2011.

[49] Y. Liu, Z. Xu, R. Feng et al., "Clinical and genetic characteristics of Chinese patients with Birt-Hogg-Dubé syndrome," Orphanet Journal of Rare Diseases, vol. 12, no. 1, p. 104, 2017.

[50] I. W. da Cunha, W. H. da Costa, M. A. Morini et al., "Expanding morphological and clinical aspects of hereditary leiomyomatosis and renal cell carcinoma (HLRCC): a case report in a patient with unusual morphology and clinical presentation," Virchows Archiv, vol. 473, no. 6, pp. 775-779, 2018.

[51] K. S. Price, A. Svenson, E. King, K. Ready, and G. A. Lazarin, "Inherited cancer in the age of next-generation sequencing," Biological Research For Nursing, vol. 20, no. 2, pp. 192-204, 2018.

[52] J. Kim, W.-Y. Park, N. K. D. Kim et al., "Good laboratory standards for clinical next-generation sequencing cancer panel tests," Journal of Pathology and Translational Medicine, vol. 51, no. 3, pp. 191-204, 2017.

[53] E. M. Bahassi and P. J. Stambrook, "Next-generation sequencing technologies: breaking the sound barrier of human genetics," Mutagenesis, vol. 29, no. 5, pp. 303-310, 2014.

[54] S. Ambardar, R. Gupta, D. Trakroo, R. Lal, and J. Vakhlu, "High throughput sequencing: an overview of sequencing chemistry," Indian Journal of Microbiology, vol. 56, no. 4, pp. 394-404, 2016.

[55] W. M. Linehan and P. T. Spellman, "Comprehensive molecular characterization of papillary renal-cell carcinoma," New England Journal of Medicine, vol. 374, no. 2, pp. 135145, 2016.

[56] S. Lin, J.-B. Zeng, G.-X. Zhao et al., "Tuberous sclerosis complex in Chinese patients: phenotypic analysis and mutational screening of TSC1/TSC2 genes," Seizure, vol. 71, pp. 322-327, 2019.

[57] E. N. Suspitsin, G. A. Yanus, M. Y. Dorofeeva et al., "Pattern of TSC1 and TSC2 germline mutations in Russian patients with tuberous sclerosis," Journal of Human Genetics, vol. 63, no. 5, pp. 597-604, 2018.

[58] E. Nicolas, E. V. Demidova, W. Iqbal et al., "Interaction of germline variants in a family with a history of early-onset clear cell renal cell carcinoma," Molecular Genetics \& Genomic Medicine, vol. 7, no. 3, p. e556, 2019.

[59] I. Bah, S. Fahiminiya, L. R. Bégin et al., "Atypical tuberous sclerosis complex presenting as familial renal cell carcinoma with leiomyomatous stroma," The Journal of Pathology: Clinical Research, vol. 4, no. 3, pp. 167-174, 2018.

[60] Z. Kote-Jarai, D. Leongamornlert, E. Saunders et al., "BRCA2 is a moderate penetrance gene contributing to young-onset prostate cancer: implications for genetic testing in prostate cancer patients," British Journal of Cancer, vol. 105, no. 8, pp. 1230-1234, 2011.

[61] D. Leongamornlert, N. Mahmud, M. Tymrakiewicz et al., "Germline BRCA1 mutations increase prostate cancer risk," British Journal of Cancer, vol. 106, no. 10, pp. 1697-1701, 2012.

[62] A. Lim, P. Rao, and S. F. Matin, "Lynch syndrome and urologic malignancies," Current Opinion in Urology, vol. 29, no. 4, pp. 357-363, 2019.

[63] H. Goldberg, C. J. D. Wallis, Z. Klaassen, T. Chandrasekar, N. Fleshner, and A. R. Zlotta, "Lynch syndrome in urologic malignancies-what does the urologist need to know?" Urology, vol. 134, pp. 24-31, 2019.

[64] M. Dominguez-Valentin, J. R. Sampson, T. T. Seppala et al., "Cancer risks by gene, age, and gender in 6350 carriers of pathogenic mismatch repair variants: findings from the prospective lynch syndrome database," Genetics in Medicine, vol. 22, no. 1, pp. 15-25, 2020.

[65] R. J. MacInnis, G. Severi, L. Baglietto et al., "Populationbased estimate of prostate cancer risk for carriers of the HOXB13 missense mutation G84E," PLoS One, vol. 8, no. 2, Article ID e54727, 2013. 
[66] R. Eeles and H. N. Raghallaigh, "Men with a susceptibility to prostate cancer and the role of genetic based screening," Translational Andrology and Urology, vol. 7, no. 1, pp. 61-69, 2018.

[67] J. L. Mohler, E. S. Antonarakis, A. J. Armstrong et al., "Prostate cancer, version 2.2019, NCCN clinical practice guidelines in oncology," Journal of the National Comprehensive Cancer Network, vol. 17, no. 5, pp. 479-505, 2019.

[68] P. Paulo, S. Maia, C. Pinto et al., "Targeted next generation sequencing identifies functionally deleterious germline mutations in novel genes in early-onset/familial prostate cancer," PLoS Genetics, vol. 14, no. 4, Article ID e1007355, 2018.

[69] J. T. Zhen, J. Syed, K. A. Nguyen et al., "Genetic testing for hereditary prostate cancer: current status and limitations," Cancer, vol. 124, no. 15, pp. 3105-3117, 2018.

[70] I. Heidegger, I. Tsaur, H. Borgmann et al., "Hereditary prostate cancer-primetime for genetic testing?" Cancer Treatment Reviews, vol. 81, Article ID 101927, 2019.

[71] V. N. Giri, S. E. Hegarty, C. Hyatt et al., "Germline genetic testing for inherited prostate cancer in practice: implications for genetic testing, precision therapy, and cascade testing," The Prostate, vol. 79, no. 4, pp. 333-339, 2019.

[72] L. Barber, T. Gerke, S. C. Markt et al., "Family history of breast or prostate cancer and prostate cancer risk," Clinical Cancer Research, vol. 24, no. 23, pp. 5910-5917, 2018.

[73] A. R. Solano, N. C. Liria, F. S. Jalil et al., "BRCA1 and BRCA2 mutations other than the founder alleles among Ashkenazi Jewish in the population of Argentina," Frontiers in Oncology, vol. 8, p. 323, 2018.

[74] R. Manchanda, M. Burnell, F. Gaba et al., "Attitude towards and factors affecting uptake of population-based BRCA testing in the Ashkenazi Jewish population: a cohort study," BJOG: An International Journal of Obstetrics \& Gynaecology, vol. 126, no. 6, pp. 784-794, 2019.

[75] E. N. Suspitsin, N. Y. Sherina, D. N. Ponomariova et al., "High frequency of BRCA1, but not CHEK2 or NBS1 (NBN), founder mutations in Russian ovarian cancer patients," Hereditary Cancer in Clinical Practice, vol. 7, no. 1, p. 5, 2009.

[76] A. G. Iyevleva, E. N. Suspitsin, K. Kroeze et al., "Non-founder BRCA1 mutations in Russian breast cancer patients," Cancer Letters, vol. 298, no. 2, pp. 258-263, 2010.

[77] I. Gorodetska, S. Serga, N. Levkovich et al., "The frequency of BRCA1 founder mutation c.5266dupC (5382insC) in breast cancer patients from Ukraine," Hereditary Cancer in Clinical Practice, vol. 13, no. 1, p. 19, 2015.

[78] A. Savanevich, O. Oszurek, J. Lubiński et al., "BRCA1 founder mutations compared to ovarian cancer in Belarus," Familial Cancer, vol. 13, no. 3, pp. 445-447, 2014.

[79] M. S. Anisimenko, G. A. Paul, A. E. Kozyakov et al., "The spectrum of BRCA1 gene mutations in early onset breast cancer patients from Russia," Siberian Journal of Oncology, vol. 17, no. 4, pp. 53-58, 2018.

[80] R. Janavicius, "Founder BRCA1/2 mutations in the Europe: implications for hereditary breast-ovarian cancer prevention and control," EPMA Journal, vol. 1, no. 3, pp. 397-412, 2010.

[81] S. Das, S. S. Salami, D. E. Spratt, S. D. Kaffenberger, M. F. Jacobs, and T. M. Morgan, "Bringing prostate cancer germline genetics into clinical practice," Journal of Urology, vol. 202, no. 2, pp. 223-230, 2019.

[82] D. Stoppa-Lyonnet, "The biological effects and clinical implications of BRCA mutations: where do we go from here?" European Journal of Human Genetics, vol. 24, no. 1, pp. S3-S9, 2016.
[83] C. Therkildsen, S. Ladelund, L. Smith-Hansen, L. J. Lindberg, and M. Nilbert, "Towards gene- and gender-based risk estimates in lynch syndrome; age-specific incidences for 13 extra-colorectal cancer types," British Journal of Cancer, vol. 117, no. 11, pp. 1702-1710, 2017.

[84] J. M. Carethers, "High predictability for identifying lynch syndrome via microsatellite instability testing or immunohistochemistry in all lynch-associated tumor types," Translational Cancer Research, vol. 8, no. 6, pp. S559-S563, 2019.

[85] H.-l. Wong, M. Christie, L. Gately et al., "Mismatch repair deficiency assessment by immunohistochemistry: for lynch syndrome screening and beyond," Future Oncology, vol. 14, no. 26, pp. 2725-2739, 2018

[86] V. R. Sarode and L. Robinson, "Screening for lynch syndrome by immunohistochemistry of mismatch repair proteins: significance of indeterminate result and correlation with mutational studies," Archives of Pathology \& Laboratory Medicine, vol. 143, no. 10, pp. 1225-1233, 2019.

[87] D. Huang, S. F. Matin, N. Lawrentschuk, and M. Roupret, "Systematic review: an update on the spectrum of urological malignancies in lynch syndrome," Bladder Cancer, vol. 4, no. 3, pp. 261-268, 2018.

[88] A. Phelan, A. Lopez-Beltran, R. Montironi et al., "Inherited forms of bladder cancer: a review of lynch syndrome and other inherited conditions," Future Oncology, vol. 14, no. 3, pp. 277-290, 2018.

[89] K. A. Nguyen, J. S. Syed, C. R. Espenschied et al., "Advances in the diagnosis of hereditary kidney cancer: initial results of a multigene panel test," Cancer, vol. 123, no. 22, pp. 43634371, 2017.

[90] https://www.thermofisher.com/life-science/sequencing/nextgeneration-sequencing/ion-torrent-next-generation-sequencingworkflow/ion-torrent-next-generation-sequencing-select-targets/ ampliseq-target-selection/ready-to-use-panels.html Ion AmpliSeq Ready-To-Use Next-Generation Sequencing (NGS) Research Panels, 2019.

[91] AmpliSeq for Illumina Ready-To-Use Panels, 2019, https:// emea.illumina.com/products/by-brand/ampliseq/ready-touse-panels.html.

[92] M. B. Christensen, K. Wadt, U. B. Jensen et al., "Exploring the hereditary background of renal cancer in Denmark," PLoS One, vol. 14, no. 4, Article ID e0215725, 2019.

[93] V. N. Giri, E. Obeid, S. E. Hegarty et al., "Understanding of multigene test results among males undergoing germline testing for inherited prostate cancer: implications for genetic counseling," The Prostate, vol. 78, no. 12, pp. 879-888, 2018.

[94] M. Rodríguez-Balada, B. Roig, M. Melé et al., "Identification of germline pathogenic variants in DNA damage repair genes by a next-generation sequencing multigene panel in BRCAX patients," Clinical Biochemistry, vol. 76, pp. 17-23, 2020.

[95] T. R. Rebbeck, T. M. Friebel, E. Friedman et al., "Mutational spectrum in a worldwide study of 29,700 families with BRCA1 or BRCA2 mutations," Human Mutation, vol. 39, no. 5, pp. 593-620, 2018.

[96] T. K. K. Mamidi, J. Wu, and C. Hicks, "Integrating germline and somatic variation information using genomic data for the discovery of biomarkers in prostate cancer," $B M C$ Cancer, vol. 19, no. 1, p. 229, 2019.

[97] L. Valle, E. Vilar, S. V. Tavtigian, and E. M. Stoffel, "Genetic predisposition to colorectal cancer: syndromes, genes, classification of genetic variants and implications for precision medicine," The Journal of Pathology, vol. 247, no. 5, pp. 574-588, 2019. 
[98] C. Van Marcke, R. Helaers, A. De Leener et al., "Tumor sequencing is useful to refine the analysis of germline variants in unexplained high-risk breast cancer families," Breast Cancer Research, vol. 22, no. 1, p. 36, 2020.

[99] C. Lu, M. Xie, M. C. Wendl et al., "Patterns and functional implications of rare germline variants across 12 cancer types," Nature Communications, vol. 6, no. 1, Article ID 10086, 2015.

[100] D. Bouvet, S. Bodo, A. Munier et al., "Methylation tolerancebased functional assay to assess variants of unknown significance in the $M L H 1$ and MSH2 genes and identify patients with lynch syndrome," Gastroenterology, vol. 157, no. 2, pp. 421-431, 2019.

[101] S. Sorscher and S. Ramkissoon, "Next-generation sequencing in order to better characterize a BRCA variant of uncertain significance," Case Reports in Oncology, vol. 10, no. 2, pp. 634-637, 2017.

[102] M. K. Ndugga-Kabuye and R. B. Issaka, "Inequities in multigene hereditary cancer testing: lower diagnostic yield and higher VUS rate in individuals who identify as hispanic, African or Asian and Pacific Islander as compared to European," Familial Cancer, vol. 18, no. 4, pp. 465-469, 2019. 